Notes on generalized $(\alpha, \beta)$-derivations in prime rings

\author{
Öznur Gölbasi
}




\title{
NOTES ON GENERALIZED $(\alpha, \beta)$-DERIVATIONS IN PRIME RINGS
}

\author{
ÖZNUR GÖLBASI
}

Received 11 November, 2004

\begin{abstract}
Let $R$ be a prime ring with characteristics different from two, $\alpha, \beta \in \operatorname{Aut}(R)$, and $I$ a nonzero ideal of $R$. Let $d: R \rightarrow R$ be a $(\alpha, \beta)$-derivation and $f: R \rightarrow R$ be generalized $(\alpha, \beta)$ derivation associated with $d$. In this case: (i) if $a f(x)=0(f(x) a=0)$ for all $x \in I$, then $a=0$; (ii) if $\beta d=d \beta$ and $[f(x), a]=0$ for all $x \in I$, then $a \in Z$ or $d(a)=0$; (iii) if $f$ acts as an endomorphism or anti-homomorphism on $I$, then $d=0$; (iv) if $\beta d=d \beta$ and $f(x y)=f(y x)$ for all $x, y \in I$ then $R$ is a commutative ring; (v) if $f$ satisfies $f\left(x^{2}\right)=f(x) \alpha(x)+\beta(x) d(x)$ for all $x \in I$, then $f(x y)=f(x) \alpha(y)+\beta(x) d(y)$ for all $x, y \in I$.
\end{abstract}

1991 Mathematics Subject Classification: 16W25, 16N60

Keywords: generalized derivation, $(\alpha, \beta)$-derivation, Jordan derivation

\section{INTRODUCTION}

The notion of a generalized derivation of a prime ring $R$ was introduced by M. Brešar [6] and B. Hvala [10]. An additive map $f$ of an associative ring $R$ is called a generalized derivation if there is a derivation $d$ of $R$ such that

$$
f(x y)=f(x) y+x d(y) \text { for all } x, y \in R .
$$

A similar notion was used by A. Nakajima [12] and N. Argaç and E. Albaş [1], who established a series of categorical properties.

As a well-known result proved by I. N. Herstein in [9, Theorem] states that if $R$ is a prime ring of characteristics different from two and if $d$ is a nonzero derivation of $R$ such that $[d(x), a]=0$ for all $x \in R$, then either $R$ is commutative or $a \in Z$. Later on, the authors extended this result to $(\sigma, \tau)$-derivation in [3]. In [8], Jui-Chi Chang showed that several results hold for any generalized $(\alpha, \beta)$-derivation of a prime ring. The purpose of this note is to generalize these theorems for a nonzero ideal of prime ring and generalized $(\alpha, \beta)$-derivation of $R$.

In [4], H. E. Bell and L. C. Kappe proved that $d$ is a derivation of $R$ which is either an endomorphism or anti-endomorphism in semi-prime ring $R$ or nonzero right ideal of $R$, then $d=0$. In view of this result, it is natural to question whether this result is true for generalized $(\alpha, \beta)$-derivations of a prime ring. Our second aim in this paper 
is to show that the above mentioned result holds for generalized $(\alpha, \beta)$-derivation of a prime ring.

In [7], M. Brešar and J. Vukman proved that every $(\alpha, \beta)$-Jordan derivation on a prime ring with characteristics different from two is a $(\alpha, \beta)$-derivation. We showed that this result holds for generalized $(\alpha, \beta)$-derivations on a nonzero ideal of prime ring $R$.

Throughout this paper, $R$ will be a prime ring with char $R \neq 2, I$ a nonzero ideal of $R, Z$ the center of $R$. Let $\alpha$ and $\beta$ be two automorphisms of $R$ into itself. For any two elements $x, y$ of $R$, we denote $[x, y]=x y-y x$ and make extensive use of the basic commutator identity $[x y, z]=x[y, z]+[x, z] y$.

\section{RESULTS}

Definition 1 ([8, Definition 1]). Let $R$ be a ring, $\alpha$ and $\beta$ automorphisms of $R$ and $d$ a $(\alpha, \beta)$-derivation of $R$. An additive mapping $f: R \rightarrow R$ is said to be right generalized $(\alpha, \beta)$-derivation associated with $d$ if

$$
f(x y)=f(x) \alpha(y)+\beta(x) d(y) \text { for all } x, y \in R .
$$

and $f$ is said to be left generalized $(\alpha, \beta)$-derivation associated with $d$ if

$$
f(x y)=d(x) \alpha(y)+\beta(x) f(y) \text { for all } x, y \in R .
$$

$f$ is said to be a generalized $(\alpha, \beta)$-derivation associated with $d$ if it is both a left and right generalized $(\alpha, \beta)$-derivation associated with $d$.

Lemma 1 ([8, Lemma 4]). Let $f \neq 0$ be a generalized $(\alpha, \beta)$-derivation of prime ring $R$ associated with $d$ and $a \in R$.

(i) If af $(x)=0$ for all $x \in R$, then $a=0$.

(ii) If $f(x) a=0$ for all $x \in R$, then $a=0$.

Lemma 2. Let $R$ be a prime ring with characteristics not equal to two, I a nonzero ideal of $R$ and $f: R \rightarrow R$ be a generalized $(\alpha, \beta)$-derivation of prime ring $R$ associated with $d$. If $f(x)=0$ for all $x \in I$, then $f=0$.

Proof. For all $x, y \in I$

$$
0=f(x y)=f(x) \alpha(y)+\beta(x) d(y)
$$

and so,

$$
I \beta^{-1}(d(y))=0 \text { for all } y \in I .
$$

Since $R$ is a prime ring, we get $d(y)=0$, for all $y \in I$. Hence, by the hypothesis, $0=f(x y)=f(x) \alpha(y)$ for all $x \in I, r \in R$. This yields that $f=0$.

Lemma 3. Let $f \neq 0$ be a generalized $(\alpha, \beta)$-derivation of prime ring $R$ associated with nonzero $(\alpha, \beta)$-derivation $d, I$ a nonzero ideal of $R$ and $a \in R$.

(i) If af $(x)=0$ for all $x \in I$, then $a=0$.

(ii) If $f(x) a=0$ for all $x \in I$, then $a=0$. 
Proof. (i) For any $x \in I, r \in R$,

$$
0=a f(x r)=a f(x) \alpha(R)+a \beta(x) d(R)
$$

and so,

$$
\beta^{-1}(a) I \beta^{-1}(d(R))=0 .
$$

Since $I$ is a nonzero ideal of prime ring $R$ and $d \neq 0$, we get $a=0$.

(ii) Similarly.

Theorem 1. Let $R$ be a prime ring with characteristics not two, $I$ a nonzero ideal of $R$ and $f: R \rightarrow R$ be a generalized $(\alpha, \beta)$-derivation of prime ring $R$ associated with nonzero $(\alpha, \beta)$-derivation $d$. If $f(x) \in Z$ for all $x \in I$, then $R$ is a commutative ring.

Proof. By the hypothesis for all $x, y \in I$,

$$
\begin{aligned}
0 & =[\alpha(y), f(x y)]=[\alpha(y), f(x) \alpha(y)+\beta(x) d(y)] \\
& =\beta(x)[\alpha(y), d(y)]+[\alpha(y), \beta(x)] d(y) \\
& =\beta(x) \alpha(y) d(y)-\beta(x) d(y) \alpha(y)+\alpha(y) \beta(x) d(y)-\beta(x) \alpha(y) d(y)
\end{aligned}
$$

and so,

$$
\alpha(y) \beta(x) d(y)-\beta(x) d(y) \alpha(y)=0 \text { for all } x, y \in I .
$$

Writing $x z, z \in I$ by $x$ in (2.3) and using this equation, we have

$$
\begin{aligned}
0 & =\alpha(y) \beta(x) \beta(z) d(y)-\beta(x) \beta(z) d(y) \alpha(y) \\
& =\alpha(y) \beta(x) \beta(z) d(y)-\beta(x) \alpha(y) \beta(z) d(y) \\
& =[\alpha(y), \beta(x)] \beta(z) d(y) .
\end{aligned}
$$

Using $I$ a nonzero ideal of prime ring $R$ and $d \neq 0$, one can obtain that $R$ is a commutative ring.

Theorem 2. Let $R$ be a prime ring with characteristics not two, $I$ a nonzero ideal of $R, d$ be a nonzero $(\alpha, \beta)$-derivation such that $d \beta=\beta d$ and $f: R \rightarrow R$ be a generalized $(\alpha, \beta)$-derivation of prime ring $R$ associated with $d$ and $a \in R$. If $[f(x), a]=0$ for all $x \in I$, then $a \in Z$ or $d(a)=0$.

Proof. By the hypothesis for all $x \in R, y \in I$, we get

$$
\begin{aligned}
0= & {[f(x y), a]=[d(x) \alpha(y)+\beta(x) f(y), a] } \\
= & d(x)[\alpha(y), a]+[d(x), a] \alpha(y)+[\beta(x), a] f(y) \\
= & d(x) \alpha(y) a-d(x) a \alpha(y)+d(x) a \alpha(y)-a d(x) \alpha(y) \\
& \quad+\beta(x) a f(y)-a \beta(x) f(y)
\end{aligned}
$$

and so,

$$
d(x) \alpha(y) a-a d(x) \alpha(y)+\beta(x) a f(y)-a \beta(x) f(y)=0
$$


for all $x \in R, y \in I$. Writing $y z, z \in R$ by $y$ in (2.4) and using (2.4), we get

$$
\begin{aligned}
0=d & (x) \alpha(y) \alpha(z) a-a d(x) \alpha(y) \alpha(z) \\
& +\beta(x) a f(y) \alpha(z)+\beta(x) a \beta(y) d(z)- \\
& -a \beta(x) f(y) \alpha(z)-a \beta(x) \beta(y) d(z) \\
= & d(x) \alpha(y) \alpha(z) a-a d(x) \alpha(y) \alpha(z)+(\beta(x) a f(y)- \\
& -a \beta(x) f(y)) \alpha(z)+\beta(x) a \beta(y) d(z)-a \beta(x) \beta(y) d(z) \\
= & d(x) \alpha(y) \alpha(z) a-d(x) \alpha(y) a \alpha(z)+\beta(x) a \beta(y) d(z) \\
& -a \beta(x) \beta(y) d(z)
\end{aligned}
$$

and so,

$$
d(x) \alpha(y)[\alpha(z), a]+[\beta(x), a] \beta(y) d(z)=0
$$

for all $x, z \in R, y \in I$. Replacing $x$ by $\beta^{-1}(a)$ in (2.5), we have

$$
d\left(\beta^{-1}(a)\right) \alpha(y)[\alpha(z), a]=0, \text { for all } z \in R, y \in I .
$$

Since $I$ is a nonzero ideal of prime ring $R$ and $d \beta=\beta d$, we get $d(a)=0$ or $a \in$ $Z$.

Theorem 3. Let $R$ be a prime ring with characteristics not two, I a nonzero ideal of $R, d$ be a nonzero $(\alpha, \beta)$-derivation such that $d \beta=\beta d$ and $f: R \rightarrow R$ be a generalized $(\alpha, \beta)$-derivation of prime ring $R$ associated with d. If $[f(x), f(y)]=0$ for all $x, y \in I$, then $R$ is a commutative ring or $d f=0$.

Proof. By Theorem 2, we have $f(I) \subset Z$ or $d f=0$. The proof is completed by Theorem 1.

Theorem 4. Let $R$ be a prime ring with characteristics not two, I a nonzero ideal of $R$ and $f: R \rightarrow R$ be a generalized $(\alpha, \beta)$-derivation of prime ring $R$ associated with $d$. If $f(x y)=f(x) f(y)$ for all $x, y \in I$, then $d=0$.

Proof. For any $x, y \in I$,

$$
f(x y)=f(x) \alpha(y)+\beta(x) d(y)=f(x) f(y), \text { for all } x, y \in I .
$$

Writing $x w, w \in I$ for $x$ in (2.6), we have

$$
f(x w) \alpha(y)+\beta(x w) d(y)=f(x w) f(y)
$$

Since $d$ acts as a homomorphism on $I$, we get

$$
\begin{array}{r}
f(x) f(w) \alpha(y)+\beta(x w) d(y)=f(x) f(w) f(y)=f(x) f(w y) \\
=f(x) f(w) \alpha(y)+f(x) \beta(w) d(y)
\end{array}
$$

and so, we have $\beta(x) \beta(w) d(y)=f(x) \beta(w) d(y)$, i. e.,

$$
(\beta(x)-f(x)) \beta(w) d(y)=0, \text { for all } x, y \in I .
$$


Since $I$ a nonzero ideal of prime ring $R$ and $\beta$ an automorphism of $R$, we conclude that

$$
f(x)=\beta(x), \text { for all } x \in I \text { or } d(y)=0, \text { for all } y \in I .
$$

Let us assume that $f(x)=\beta(x)$, for all $x \in I$. Replacing $x$ by $x y, y \in I$, we have $f(x y)=\beta(x y)$ and $d(x) \alpha(y)+\beta(x) f(y)=\beta(x) \beta(y)=\beta(x) f(y)$.

Thus, we obtain that $d(x)=0$ for all $x \in I$ for any cases. That is $d=0$. This completes the proof.

Theorem 5. Let $R$ be a prime ring with characteristics not two, I a nonzero ideal of $R$ and $f: R \rightarrow R$ be a generalized $(\alpha, \beta)$-derivation of prime ring $R$ associated with $d$. If $f(x y)=f(y) f(x)$ for all $x, y \in I$, then $d=0$.

Proof. Since $d$ acts as an anti-homorphism on $I$, we get

$$
f(x y)=d(x) \alpha(y)+\beta(x) f(y)=f(y) f(x), \text { for all } x, y \in I .
$$

Replacing $y$ by $x y, y \in I$ in (2.7), we obtain $d(x) \alpha(x y)+\beta(x) f(x y)=f(x y) f(x)$,

$$
d(x) \alpha(x y)+\beta(x) f(y) f(x)=d(x) \alpha(y) f(x)+\beta(x) f(y) f(x)
$$

and so,

$$
d(x) \alpha(x) \alpha(y)=d(x) \alpha(y) f(x), \text { for } x, y \in I .
$$

Substituting $y w, w \in I$ for $y$ in this equation and using (2.8), we get

$$
\begin{aligned}
0 & =d(x) \alpha(x) \alpha(y) \alpha(w)-d(x) \alpha(y) \alpha(w) f(x) \\
& =d(x) \alpha(y) f(x) \alpha(w)-d(x) \alpha(y) \alpha(w) f(x) \\
& =d(x) \alpha(y)[f(x), \alpha(w)] .
\end{aligned}
$$

Since $\alpha$ is an automorphism and $I$ a nonzero ideal of prime ring $R$, we obtain

$$
d(x)=0 \text { or }[f(x), \alpha(w)]=0, \text { for all } x, w \in I .
$$

Now let us define $A=\{x \in I \mid d(x)=0\}$ and $B=\{x \in I \mid[f(x), \alpha(w)]=0$, for all $w \in I\}$. Clearly each $A$ and $B$ is an additive subgroup of $I$. Moreover, $I$ is the set theoretic union of $A$ and $B$. But a group cannot be the set theoretic union of two proper subgroups, hence $A=I$ or $B=I$. In the former case, we have $d(R)=0$, which completes the proof. If $B=I$, then we get

$$
0=[f(x), \alpha(w r)]=\alpha(w)[f(x), \alpha(R)]
$$

for all $x, w \in I, r \in R$.

Thus, we obtain that $f(I) \subset Z$. Since $f$ acts as an endomorphism of $R$, it follows that $d=0$, via Theorem 4 .

Theorem 6. Let $R$ be a prime ring with characteristics not two, $I$ a nonzero ideal of $R$ and $f: R \rightarrow R$ be a generalized $(\alpha, \beta)$-derivation of prime ring $R$ associated with nonzero $(\alpha, \beta)$-derivation $d$. If $\beta d=d \beta$ and $f(x y)=f(y x)$ for all $x, y \in I$, then $R$ is a commutative ring. 
Proof. Let $c \in I$ be a constant element such that $f(c)=0$ (i. e., $[x, y])$ and $z$ be an arbitrary element of $I$. The condition that $f(c z)=f(z c)$ yields that $f(c) \alpha(z)+$ $\beta(c) d(z)=d(z) \alpha(c)+\beta(z) f(c)$ and so,

$$
\beta(c) d(z)=d(z) \alpha(c), \text { for } z \in I .
$$

Thus $[d(z), c]_{\alpha, \beta}=0$, for all $z \in I$. Replacing $z$ by $w z, w \in I$ and using this equation, we have

$$
\begin{aligned}
0 & =[d(w z), c]_{\alpha, \beta}=[d(w) \alpha(z)+\beta(w) d(z), c]_{\alpha, \beta} \\
& =d(w) \alpha([z, c])+[d(w), c]_{\alpha, \beta} \alpha(z)+\beta(w)[d(z), c]_{\alpha, \beta}+\beta([w, c]) d(z)
\end{aligned}
$$

and so,

$$
d(w) \alpha([z, c])+\beta([w, c]) d(z)=0, \text { for all } w, z \in I .
$$

Writing $c$ (since $c \in I$ ) by $z$ in (2.9), we have $\beta([w, c]) d(c)=0$.This gives us $[w, c] \beta^{-1}(d(c))=0$, for all $w \in I$, all $c \in I$ such that $f(c)=0$. If we take $r w, r \in$ $R, w \in I$ instead of $w$ in this equation, we have

$$
[r, c] w \beta^{-1}(d(c))=0, \text { for all } r \in R .
$$

Since $I$ is a nonzero ideal of prime ring $R$, we obtain

$$
c \in Z \text { or } d(c)=0 \text {, for all } c \in I \text { such that } f(c)=0 .
$$

So, we obtain

$$
[x, y] \in Z \text { or } d([x, y])=0, \text { for all } x, y \in I .
$$

because of $f([x, y])=0$, for all $x, y \in I$. If $d([x, y])=0$, for all $y \in I\}$.

This shows that additive group $I$ is the union of two of its additive subgroups $A=\{x \in I \mid d([x, y])=0$, for all $y \in I\}$ and $B=\{x \in I \mid[x, y] \in Z$, for all $y \in I\}$.

If $I=A$, then $R$ is a commutative ring (i. e., $[x, y] \in Z$, for all $x, y \in I$ ) by [2, Theorem 3]. So, we can take $I=B$ and $[x, y] \in Z$, for all $x, y \in I$. Let us define an inner derivation $I_{x}: R \rightarrow R, I_{x}^{2}(y)=0$, for all $y \in I$. This shows $I \subset Z$ and so $R$ is a commutative ring by [5, Theorem 1$]$.

Definition 2. Let $R$ be a ring, $\beta$ and $\alpha$ automorphisms of $R$ and $d$ a $(\alpha, \beta)$ derivation of $R$. An additive mapping $f: R \rightarrow R$ is said to be right generalized $(\alpha, \beta)$-Jordan derivation associated with $d$ if

$$
f\left(x^{2}\right)=f(x) \alpha(x)+\beta(x) d(x) \text { for all } x \in R .
$$

and $f$ is said to be left generalized $(\alpha, \beta)$-Jordan derivation associated with $d$ if

$$
f\left(x^{2}\right)=d(x) \alpha(x)+\beta(x) f(x) \text { for all } x \in R .
$$

$f$ is said to be a generalized $(\alpha, \beta)$-Jordan derivation associated with $d$ if it is both a left and right generalized $(\alpha, \beta)$-Jordan derivation associated with $d$. 
Lemma 4. Let $f: R \rightarrow R$ be a right generalized $(\alpha, \beta)$-Jordan derivation on $I$. For all $x, y, z \in I$,

$$
\begin{aligned}
f(x y+y x)=f & (x) \alpha(y)+\beta(x) d(y)+f(y) \alpha(x)+\beta(y) d(x) \\
f(x y x)=f & (x) \alpha(y x)+\beta(x) d(y) \alpha(x)+\beta(x y) d(x) \\
f(x y z+z y x)= & f(x) \alpha(y z)+\beta(x) d(y) \alpha(z)+\beta(x y) d(z) \\
& +f(z) \alpha(y x)+\beta(z) d(y) \alpha(x)+\beta(z y) d(x) \\
& +f(z) \alpha(y x)+\beta(z) d(y) \alpha(x)+\beta(z y) d(x) .
\end{aligned}
$$

Proof. Assertion (1). Linearizing (2.10), we get

$$
\begin{aligned}
f\left((x+y)^{2}\right)= & f\left((x+y)(x+y)=f\left(x^{2}+x y+y x+y^{2}\right)\right. \\
= & f\left(x^{2}\right)+f(x y+y x)+f\left(y^{2}\right) \\
= & f(x) \alpha(x)+\beta(x) d(x) \\
& \quad+f(x y+y x)+f(y) \alpha(y)+\beta(y) d(y)
\end{aligned}
$$

for all $x, y \in I$. On the other hand,

$$
\begin{aligned}
f\left((x+y)^{2}\right) & =f(x+y) \alpha(x+y)+\beta(x+y) d(x+y) \\
& =f(x) \alpha(x)+f(y) \alpha(x)+f(x) \alpha(y)+f(y) \alpha(y) \\
& +\beta(x) d(x)++\beta(x) d(y)+\beta(y) d(x)+\beta(y) d(y)
\end{aligned}
$$

for all $x, y \in I$. Comparing (2.12) and (2.13), we have

$$
f(x y+y x)=f(x) \alpha(y)+\beta(x) d(y)+f(y) \alpha(x)+\beta(y) d(x)
$$

for all $x, y \in I$.

Assertion (2). Replacing $y$ by $x y+y x$ in (1), we get

$$
\begin{aligned}
f(x(x y+y x)+(x y+y x) x)=f\left(x^{2} y+x y x+x y x+y x^{2}\right) \\
=f\left(x^{2} y+y x^{2}\right)+2 f(x y x) \\
=f\left(x^{2}\right) \alpha(y)+\beta\left(x^{2}\right) d(y)+f(y) \alpha\left(x^{2}\right) \\
\quad+\beta(y) d\left(x^{2}\right)+2 f(x y x) \\
=f(x) \alpha(x y)+\beta(x) d(x) \alpha(y)+\beta\left(x^{2}\right) d(y) \\
\quad+f(y) \alpha\left(x^{2}\right)+\beta(y) d(x) \alpha(x) \\
\quad+\beta(y x) d(x)+2 f(x y x)
\end{aligned}
$$


for all $x, y \in I$. On the other hand, we have

$$
\begin{aligned}
f(x(x y+y x)+(x y+y x) x)=f(x) \alpha(x y+y x)+\beta(x) d(x y+y x) \\
\quad+f(x y+y x) \alpha(x)+\beta(x y+y x) d(x) \\
=f(x) \alpha(x y)+f(x) \alpha(y x)+\beta(x) d(x) \alpha(y)+\beta\left(x^{2}\right) d(y) \\
\quad+\beta(x) d(y) \alpha(x)+\beta(x y) d(x)+f(x) \alpha(y x) \\
+\beta(x) d(y) \alpha(x)+f(y) \alpha\left(x^{2}\right)+\beta(y) d(x) \alpha(x) \\
+\beta(x y) d(x)+\beta(y x) d(x)
\end{aligned}
$$

for all $x, y \in I$. Comparing (2.14) and (2.15) and using char $R \neq 2$, we get the required result.

Assertion (3). Linearizing (2) on $x$, we get

$$
\begin{aligned}
f((x+z) y(x+y))= & f(x y x+x y z+z y x+z y z) \\
= & f(x y x)+f(x y z+z y x)+f(z y z) \\
= & f(x) \alpha(y x)+\beta(x) d(y) \alpha(x)+\beta(x y) d(x) \\
& \quad+f(x y z+z y x)+f(z) \alpha(y z) \\
& \quad+\beta(z) d(y) \alpha(z)+\beta(z y) d(z)
\end{aligned}
$$

for all $x, y, z \in I$. Computing now $f((x+z) y(x+z))$ in another way, we get

$$
\begin{aligned}
f((x+z) y(x+z))=f & (x+z) \alpha(y x+y z)+\beta(x+z) d(y) \alpha(x+z) \\
& +\beta(x y+z y) d(x+z) \\
= & f(x) \alpha(y x)+f(x) \alpha(y z)+f(z) \alpha(y x)+f(z) \alpha(y z) \\
& +\beta(x) d(y) \alpha(x)+\beta(x) d(y) \alpha(z) \\
& +\beta(z) d(y) \alpha(x)+\beta(z) d(y) \alpha(z) \\
& +\beta(x y) d(x)+\beta(x y) d(z)+\beta(z y) d(x) \\
& +\beta(z y) d(z)
\end{aligned}
$$

for all $x, y, z \in I$. Comparing (2.16) and (2.17), we have

$$
\begin{array}{r}
f(x y z+z y x)=f(x) \alpha(y z)+\beta(x) d(y) \alpha(z)+\beta(x y) d(z)+f(z) \alpha(y x) \\
+\beta(z) d(y) \alpha(x)+\beta(z y) d(x)
\end{array}
$$

for all $x, y, z \in I$.

We introduce the abbreviation

$$
x^{y}=f(x y)-f(x) \alpha(y)-\beta(x) d(y)
$$

for all $x, y \in I$. 
Observe that, by Lemma 4 (1), we have $f(x y+y x)=f(x) \alpha(y)+\beta(x) d(y)+$ $f(y) \alpha(x)+\beta(y) d(x)$ and so, $f(x y)-f(x) \alpha(y)-\beta(x) d(y)=-(f(y x)-$ $f(y) \alpha(x)-\beta(y) d(x))$. That is,

$$
x^{y}=-y^{x} \text { for all } x, y \in I .
$$

Lemma 5. Let $f: R \rightarrow R$ be a right generalized $(\alpha, \beta)$-Jordan derivation on $I$. For all $x, y \in I$,

$$
x^{y}[\alpha(x), \alpha(y)]=0 .
$$

Proof. Replacing $z$ by $x y$ in Lemma 4 (3), we get

$$
\begin{aligned}
f\left((x y)^{2}+x y^{2} x\right)=f & \left((x y)^{2}\right)+f\left(x y^{2} x\right)=f(x y) \alpha(x y)+\beta(x y) d(x y) \\
& +f(x) \alpha\left(x^{2} y\right)+\beta(x) d\left(y^{2}\right) \alpha(x)+\beta\left(x y^{2}\right) d(x) \\
=f & (x y) \alpha(x y)+\beta(x y) d(x) \alpha(y)+\beta(x y x) d(y) \\
& +f(x) \alpha\left(x^{2} y\right)+\beta(x) d(y) \alpha(y x) \\
& +\beta(x y) d(y) \alpha(x)+\beta\left(x y^{2}\right) d(x)
\end{aligned}
$$

for all $x, y \in I$. On the other hand, we get

$$
\begin{gathered}
f(x y(x y)+(x y) y x)=f(x) \alpha(y x y)+\beta(x) d(y) \alpha(x y)+\beta(x y) d(x y) \\
\quad+f(x y) \alpha(y x)+\beta(x y) d(y) \alpha(x)+\beta\left(x y^{2}\right) d(x) \\
=f(x) \alpha(y x y)+\beta(x) d(y) \alpha(x y)+\beta(x y) d(x) \alpha(y) \\
\quad+\beta(x y x) d(y)+f(x y) \alpha(y x)+\beta(x y) d(y) \alpha(x) \\
+\beta\left(x y^{2}\right) d(x)
\end{gathered}
$$

for all $x, y \in I$. Comparing these two equations, we have

$$
\begin{aligned}
& f(x y) \alpha(x y)+f(x) \alpha\left(y^{2} x\right)+\beta(x) d(y) \alpha(y x) \\
& \quad=f(x) \alpha(y x y)+\beta(x) d(y) \alpha(x y)+f(x y) \alpha(y x) .
\end{aligned}
$$

That is, $x^{y}[\alpha(x), \alpha(y)]=0$ for all $x, y \in I$.

Theorem 7. Let $R$ be a non-commutative prime ring with characteristics not two, $I$ a nonzero ideal of $R$. If $f$ be a right generalized $(\alpha, \beta)$-Jordan derivation on $I$, then $f$ is generalized $(\alpha, \beta)$-derivation on I.

Proof. From Lemma 4 (3), we have

$$
\begin{aligned}
f(x w y+y w x)=f(x) & \alpha(w y)+\beta(x) d(w) \alpha(y)+\beta(x w) d(y) \\
& +f(y) \alpha(w x)+\beta(y) d(w) \alpha(x)+\beta(y w) d(x)
\end{aligned}
$$


for all $x, y, w \in I$. Replacing $x$ by $x y$ and $y$ by $y x$ in (2.21), we get

$$
\begin{aligned}
f((x y) & w(y x)+(y x) w(x y))=f(x y) \alpha(w y x)+\beta(x y) d(w) \alpha(y x) \\
& +\beta(x y w) d(y x)+f(y x) \alpha(w x y) \\
& +\beta(y x) d(w) \alpha(x y)+\beta(y x w) d(x y) \\
=f & (x y) \alpha(w y x)+\beta(x y) d(w) \alpha(y x)+\beta(x y w) d(y) \alpha(x) \\
& +\beta(x y w y) d(x)+f(y x) \alpha(w x y)+\beta(y x) d(w) \alpha(x y) \\
& +\beta(y x w) d(x) \alpha(y)+\beta(y x w x) d(y)
\end{aligned}
$$

for all $x, y, w \in I$. On the other hand, we have

$$
\begin{array}{r}
f((x y) w(y x)+(y x) w(x y))=f(x(y w y) x)+f(y(x w x) y) \\
=f(x) \alpha(y w y x)+\beta(x) d(y w y) \alpha(x)+\beta(x y w y) d(x) \\
+f(y) \alpha(x w x y)+\beta(y) d(x w x) \alpha(y)+\beta(y x w x) d(y) \\
=f(x) \alpha(y w y x)+\beta(x) d(y) \alpha(w y x)+\beta(x y) d(w) \alpha(y x) \\
+\beta(x y w) d(y) \alpha(x)+\beta(x y w y) d(x)+f(y) \alpha(x w x y) \\
+\beta(y) d(x) \alpha(w x y)+\beta(y x) d(w) \alpha(x y) \\
+\beta(y x w) d(x) \alpha(y)+\beta(y x w x) d(y)
\end{array}
$$

for all $x, y, w \in I$. Comparing equations (2.22) and (2.23), we obtain

$$
\begin{aligned}
\{f(y x)-f(y) \alpha(x)-\beta(y) d(x)\} \alpha(w) \alpha(x y) & \\
& +\{f(x y)-f(x) \alpha(y)-\beta(x) d(y)\} \alpha(w) \alpha(y x)=0
\end{aligned}
$$

and hence

$$
y^{x} \alpha(w) \alpha(x y)+x^{y} \alpha(w) \alpha(y x)=0
$$

for all $x, y, w \in I$. Using (2.18), we get $x^{y} \alpha(I) \alpha([y, x])=0$ for all $x, y \in I$. Since $I$ is a nonzero ideal of prime ring $R$, we obtain for each pair $x, y \in I$ either $x^{y}=0$ or $[x, y]=0$. Notice that the mappings $(x, y) \rightarrow x^{y}$ and $(x, y) \rightarrow[x, y]$ satisfy the requirements of [5, Lemma 4]. Hence $x^{y}=0$ for all $x, y \in I$ or $[x, y]^{2}=0$ for all $x, y \in I$. If $[x, y]^{2}=0$ for all $x, y \in I$, then for each $x \in I, I_{x}(y)^{2}=0$, for all $y \in I$, where $I_{x}$ is the inner derivation. This yields that $R$ is commutative, a contradiction by [11, Theorem 3]. Thus, we have $x^{y}=0$ for all $x, y \in I$. This completes the proof.

\section{REFERENCES}

[1] N. Argaç and E. Albaş, "On generalized $(\sigma, \tau)$-derivations," Sibirsk. Mat. Zh., vol. 43, no. 6, pp. 1211-1221, 2002.

[2] M. Ashraf and N. ur Rehman, "On $(\sigma, \tau)$-derivations in prime rings," Arch. Math. (Brno), vol. 38, no. 4, pp. 259-264, 2002.

[3] N. Aydın and K. Kaya, "Some generalizations in prime rings with $(\sigma, \tau)$-derivation," Doğa Mat., vol. 16, no. 3, pp. 169-176, 1992. 
[4] H. E. Bell and L.-C. Kappe, "Rings in which derivations satisfy certain algebraic conditions," Acta Math. Hungar., vol. 53, no. 3-4, pp. 339-346, 1989.

[5] J. Bergen, I. N. Herstein, and J. W. Kerr, "Lie ideals and derivations of prime rings," J. Algebra, vol. 71, no. 1, pp. 259-267, 1981.

[6] M. Brešar and J. Vukman, "Jordan $(\Theta, \phi)$-derivations," Glas. Mat. Ser. III, vol. 26(46), no. 1-2, pp. 13-17, 1991.

[7] M. Brešar, "On the distance of the composition of two derivations to the generalized derivations," Glasgow Math. J., vol. 33, no. 1, pp. 89-93, 1991.

[8] J.-C. Chang, "On the identity $h(x)=a f(x)+g(x) b$," Taiwanese J. Math., vol. 7, no. 1, pp. 103-113, 2003.

[9] I. N. Herstein, "A note on derivations. II," Canad. Math. Bull., vol. 22, no. 4, pp. 509-511, 1979.

[10] B. Hvala, "Generalized derivations in rings," Comm. Algebra, vol. 26, no. 4, pp. 1147-1166, 1998.

[11] P. H. Lee and T. K. Lee, "On derivations of prime rings," Chinese J. Math., vol. 9, no. 2, pp. $107-110,1981$.

[12] A. Nakajima, "On categorical properties of generalized derivations," Sci. Math., vol. 2, no. 3, pp. 345-352, 1999.

\section{Author's address}

\section{Öznur Gölbasi}

Cumhuriyet University, Faculty of Arts and Science, Department of Mathematics, Sivas, Turkey

E-mail address: ogolbasi@cumhuriyet.edu.tr

URL: http://www.cumhuriyet.edu.tr 